\title{
GUIDING ISOTOPIES AND HOLOMORPHIC MOTIONS
}

\section{Frederick P. Gardiner, Yunping Jiang and Zhe Wang}

CUNY Graduate Center, Department of Mathematics

365 Fifth Avenue, New York, NY 10016, U.S.A.; frederick.gardiner@gmail.com

Queens College of CUNY, Department of Mathematics, Flushing, NY 11367 and CUNY Graduate Center, Department of Mathematics

365 Fifth Avenue, New York, NY 10016, U.S.A.; yunping.jiang@qc.cuny.edu

Bronx Community College of CUNY, Department of Mathematics and Computer Science 2155 University Avenue, Bronx, NY 10453, U.S.A.; wangzhecuny@gmail.com

\begin{abstract}
We develop an isotopy principle for holomorphic motions. Our main result concerns the extendability of a holomorphic motion $h(t, z)$ of a finite subset $E$ of the Riemann sphere $\overline{\mathbf{C}}$ parameterized by a pointed hyperbolic Riemann surface $\left(X, t_{0}\right)$. We prove that if this holomorphic motion has a guiding quasiconformal isotopy, then it can be extended to a new holomorphic motion of $E \cup\{p\}$ for any point $p$ in $\overline{\mathbf{C}} \backslash E$ that follows the guiding isotopy. The proof gives a canonical way to replace a continuous motion of the $(n+1)$-st point by a holomorphic motion while leaving unchanged the given holomorphic motion of the first $n$ points.
\end{abstract}

\section{Introduction}

Suppose we are given a finite set $\left\{p_{1}(t), \ldots, p_{n}(t)\right\}$ of holomorphic maps from a pointed hyperbolic Riemann surface $\left(X, t_{0}\right)$ with values in a Riemann surface $Y$ such that the set

$$
E_{t}=\left\{p_{1}(t), \ldots, p_{n}(t)\right\}
$$

consists of $n$ distinct points in $Y$ for every $t \in X$. Here $X$ is called the parameter space and $Y$ is called the dynamical space.

Suppose in addition we are given a continuous function $p_{n+1}(t): X \rightarrow Y$ such that for every value of $t \in X$,

$$
\widetilde{E}_{t}=E_{t} \cup\left\{p_{n+1}(t)\right\}=\left\{p_{1}(t), \ldots, p_{n}(t), p_{n+1}(t)\right\}
$$

consists of $n+1$ distinct points in $Y$.

If all of these hypotheses are satisfied, then $E_{t}$ is a holomorphic motion in $Y$ parameterized by $X$ with a continuous motion extension $\widetilde{E}_{t}$ in $Y$ parameterized by the same $X$.

doi:10.5186/aasfm.2015.4031

2010 Mathematics Subject Classification: Primary 30F60; Secondary 32G15, 30C70, 30C75.

Key words: Guiding quasiconformal isotopy, holomorphic motion, continuous motion, holomorphic quadratic differential, heights mapping.

The second author is partially supported by the collaboration grant from the Simons Foundation (No. 199837), the CUNY collaborative incentive research grant (No. 1861), awards from PSC-CUNY, and a grant from the NSF of China (No. 11171121), and a collaboration grant from Academy of Mathematics and Systems Science and the Morningside Center of Mathematics at the Chinese Academy of Sciences. 
The main goal of this paper is to give an additional condition, which we call a guiding quasiconformal isotopy condition, that provides a canonical way to replace the continuous function $p_{n+1}(t): X \rightarrow Y$ with a holomorphic function $\widehat{p}_{n+1}(t): X \rightarrow Y$ such that

$$
\widehat{E}_{t}=E_{t} \cup\left\{\widehat{p}_{n+1}(t)\right\}=\left\{p_{1}(t), \ldots, p_{n}(t), \widehat{p}_{n+1}(t)\right\}
$$

becomes a holomorphic motion extension of $E_{t}$ parameterized by the same $X$. More precisely, we need $\widehat{p}_{n+1}\left(t_{0}\right)=p_{n+1}\left(t_{0}\right)$ and $\widehat{p}_{n+1}(t) \neq p_{i}(t)$ for all $t \in X$ and $1 \leq i \leq n$

Our main theorem says that this is possible when $Y=\overline{\mathbf{C}}$, the Riemann sphere. The same method of proof works when $Y$ is equal to any Riemann surface of finite analytic type, and we plan to incorporate this generalization into a subsequent paper. One application is Slodkowski's theorem which concerns the extension of a holomorphic motion $h(t, z)$ of a subset $E$ in the Riemann sphere $\overline{\mathbf{C}}$ parameterized by the open unit disk $\Delta$ with the basepoint 0 . Our new proof has two parts. The first part is the topological part which says that any holomorphic motion of a finite subset $E$ of the Riemann sphere $\overline{\mathbf{C}}$ parameterized by the open unit disk $\Delta$ always has a guiding quasiconformal isotopy. The second part is the geometric part which is the main theorem of this paper. The new proof provides a canonical holomorphic replacement.

This result relies on the existence of a canonical cylindrical differential with a double pole at any point, on the heights mapping for quadratic differentials, and on the use of the Alhfors-Weill local holomorphic section (i.e., harmonic Beltrami differentials) on Teichmüller spaces to produce holomorphic coordinates for the Teichmüller space of quasi-circles.

The paper is organized as follows. In section 2 we review the definition of a continuous motion and define a guiding quasiconformal isotopy. In section 3 we state Theorem 1 which is the main result and Theorem 2 which is Slodkowski's theorem. In section 4 we present the theory of holomorphic quadratic differentials as solutions to extremal problems, the heights mapping theorem and a limiting process which yields a cylindrical quadratic differential with a single semi-infinite cylinder. In section 5 we define our extension that follows a given guiding quasiconformal isotopy. In section 6 we prove that the extension defined in section 5 is holomorphic. The main ideas in sections 5 and 6 concern extremal infinite cylinders corresponding to quadratic differentials with double poles and on the use of Alhfors-Weill local holomorphic section (harmonic Beltrami differentials) for Teichmüller spaces. The detailed proof of Theorem 1 is completed by sections $4,5,6$. In section 7 we give our new proof of Slodkowski's theorem. In section 8 we discuss topological obstructions that show that motions parameterized by a non simply-connected pointed hyperbolic Riemann surface do not necessarily have guiding isotopies. Thus the guiding isotopy assumption in Theorem 1 is necessary.

Acknowledgement. We have had many helpful conversations with Jeremy Kahn, Linda Keen, Sudeb Mitra, and Hiroshige Shiga.

\section{Motions and guiding isotopies}

In this section we define a motion of a set $E$ in a Riemann surface $Y$ parameterized by a pointed hyperbolic Riemann surface $\left(X, t_{0}\right)$ and guiding isotopies of the motion. 
Definition 1. (Continuous motion) Let $E \subset Y$. A continuous motion

$$
h(t, z): X \times E \rightarrow Y
$$

of $E$ in $Y$ parameterized by $X$ is a continuous map of $(t, z)$ satisfying

1) $h\left(t_{0}, p\right)=p$, for all $p \in E$, and

2) for any fixed $t \in X, h_{t}(p)=h(t, p): E \rightarrow E_{t}=h_{t}(E) \subseteq Y$ is a homeomorphism.

We think of the parameter $t \in X$ as measuring time and $h_{t}(z)$ as specifying the motion of the point $z$ in a dynamical space $Y$.

Definition 2. (Holomorphic motion) A continuous motion $h(t, z)$ of $E$ in $Y$ parameterized by $X$ is called a holomorphic motion of $E$ if for each fixed $p \in E$, $h_{p}(t)=h(t, p): X \rightarrow Y$ is holomorphic.

Definition 3. (Extension) Suppose

$$
h(t, z): X \times E \rightarrow Y \text { and } \widehat{h}(t, z): X \times \widetilde{E} \rightarrow Y
$$

are continuous (or holomorphic) motions of subsets $E$ and $\widehat{E}$ in $Y$ parameterized by $X$. If $E \subset \widehat{E}$ and $\widehat{h}(t, z)=h(t, z)$ for all $z \in E$ and all $t \in X$, then $\widehat{h}$ is called an extension of $h$ to $\widehat{E}$.

Definition 4. (Guiding quasiconformal isotopy) A guiding isotopy for a continuous motion $h(t, z)$ of $E$ in $Y$ parameterized by $X$ is an extension of $h$ to a continuous motion $G$ of the all of $Y$ parameterized by the same $X$. It is called a guiding quasiconformal isotopy if for each fixed $t \in X, G_{t}(z)=G(t, z): Y \rightarrow Y$ is a quasiconformal homeomorphism of $Y$ and if the Beltrami coefficient

$$
X \ni t \mapsto \mu_{t} \in L^{\infty}(Y)
$$

where

$$
\mu_{t}(z)=\frac{\bar{\partial} G_{t}(z)}{\partial G_{t}(z)}
$$

defines a continuous map with respect to the topology of $X$ and the $L^{\infty}$-norm topology of $L^{\infty}(Y)$.

A notion very close to the concept of guiding isotopy is the notion of quasiconformal trivializaiton defined in Definition 2.1 of [11] and Definition 1.1 of [25]. In contrast to our definition of quiding quasiconformal isotopy the definition in [11] and [25] does not require that $\mu_{t}$ vary continuously in the $L^{\infty}$ topology. The idea of using a guiding quasiconformal isotopy is suggested in [7] where it is used to solve a version of the guiding homeomorphism problem.

We have the following proposition about guiding quasiconformal isotopies.

Proposition 1. (Uniqueness up to isotopy) Suppose $h(t, z): X \times E \rightarrow Y$ is a holomorphic motion. If $h$ has a guiding quasiconformal isotopy $G(t, z): X \times Y \rightarrow Y$, then any guiding quasiconformal isotopy $H(t, z): X \times Y \rightarrow Y$ is quasiconformally isotopic to $G$ on $Y \backslash E$.

Proof. For any $t \in X$, let $H_{t}(z)=H(t, z)$ and $G_{t}(z)=G(t, z)$. Then both of them are quasiconformal homeomorphisms of $Y$. Let $F_{t}=G_{t}^{-1} \circ H_{t}$. Since both 
of them are extensions of $h$, we have that $F_{t}(z)=z$ for all $z \in E$. Since $F_{t}$ is a quasiconformal homeomorphism, we can define its Beltrami coefficient

$$
\mu_{t}(z)=\frac{\bar{\partial} F_{t}(z)}{\partial F_{t}(z)} \in L^{\infty}(Y)
$$

on $Y$. It is a continuous map from $X$ to $L^{\infty}(Y)$. Thus $\phi(t)=F_{t}$ is a continuous map from $X$ to the space of quasiconformal homeomorphisms of $Y$. But $\phi\left(t_{0}\right)=F_{t_{0}}=$ Id.

This proposition shows that the isotopy class of the extension $H$ of $h$ relative to $Y \backslash E$ is unique. A related result appeared in [25, Theorem C] as well as in [20, Proposition 5.13].

\section{Statement of the main results}

In this section we suppose $\left(X, t_{0}\right)$ is a pointed hyperbolic Riemann surface and $Y=\overline{\mathbf{C}}$. We also suppose $h(t, z)$ is a continuous motion of $E=\left\{p_{1}, \ldots, p_{n}\right\}$ in $\overline{\mathbf{C}}$ parameterized by $X$. Since the points $p_{j}(t)=h\left(t, p_{j}\right)$ of $E$ move continuously and distinctly, there is a continuous path of Möbius transformations $A_{t}$ such that $A_{t}\left(p_{1}(t)\right)=0, A_{t}\left(p_{2}(t)\right)=1$, and $A_{t}\left(p_{3}(t)\right)=\infty$. Then the ordered $n$-tuple

$$
A_{t}\left(E_{t}\right)=\left\{0,1, \infty, A_{t}\left(p_{4}(t)\right), \ldots, A_{t}\left(p_{n}(t)\right)\right\}
$$

is a continuous motion of $A_{0}(E)$ and the continuous motion $A_{t} \circ h$ is normalized at 0,1 and $\infty$. Obviously, if $h$ is a holomorphic motion, then the family $A_{t}$ also depends on $t$ holomorphically. Thus $A_{t} \circ h$ is also a holomorphic motion and so by showing how to extend a holomorphic motion of a subset in $\overline{\mathbf{C}}$ normalized at $0,1, \infty$, and by choosing an appropriate holomorphic path of Möbius transformations $A_{t}$ we also provide a method for extending holomorphic motions that are not normalized.

Thus we can assume in the beginning that $E$ contains $0,1, \infty$ and $h$ is a continuous (or holomorphic) motion normalized at $0,1, \infty$.

Theorem 1. (Main Theorem) Suppose

$$
E=\left\{p_{1}=0, p_{2}=1, p_{3}=\infty, p_{4}, \cdots, p_{n}\right\}
$$

is a finite subset of $\overline{\mathbf{C}}$ containing $n \geq 3$ distinct points. Assume $h: X \times E \rightarrow \overline{\mathbf{C}}$ is a normalized holomorphic motion of $E$ in $\overline{\mathbf{C}}$ parameterized by $X$. Suppose $p$ is a point in $\overline{\mathbf{C}} \backslash E$. If $h$ has a guiding quasiconformal isotopy $G: X \times \overline{\mathbf{C}} \rightarrow \overline{\mathbf{C}}$, then there is a holomorphic motion $\widehat{h}: X \times(E \cup\{p\}) \rightarrow \overline{\mathbf{C}}$ that extends $h$.

If the parameter space $X$ is compact or compact except for a finite number of punctures, this theorem has no interest because the only holomorphic functions are constant. So the interesting case is when $X$ is an open hyperbolic Riemann surface with a nontrivial boundary component.

We prove the Main Theorem in sections 5 and 6 .

If $X=\Delta$, the unit disc, and $t_{0}=0$, then Theorem 1 gives a new proof of the following theorem.

Theorem 2. (Slodkowski's Theorem) Any holomorphic motion $h(t, z): \Delta \times E \rightarrow$ $\overline{\mathbf{C}}$ of a subset $E$ in $\overline{\mathbf{C}}$ parameterized by $\Delta$ can be extended to a holomorphic motion $H(t, z): \Delta \times \overline{\mathbf{C}} \rightarrow \overline{\mathbf{C}}$. 
We prove in section 7 that any holomorphic motion of a finite subset in $\overline{\mathbf{C}}$ parameterized by $\Delta$ has a guiding quasiconformal isotopy. Thus when the parameter space is the unit disc the assumption of Theorem 1 is always satisfied and this yields our proof Theorem 2 .

\section{Cylinders with maximal modulus}

In this section we review basic facts about the existence of cylindrical holomorphic quadratic differentials.

Theorem 3. Assume $E$ is a finite subset of $\overline{\mathbf{C}}$ containing two or more points and $\Delta(p)$ is a conformal disc centered at $p$ in $\overline{\mathbf{C}} \backslash E$. Let $\gamma$ be a simple closed curve in $Y=\overline{\mathbf{C}} \backslash(E \cup \Delta(p))$ and homotopic to the boundary of $\Delta(p)$. Then there exists a unique holomorphic quadratic differential $q$ of finite norm defined on $Y$ with the following properties:

(1) all of the regular horizontal trajectories of $q$ are closed and homotopic to $\gamma$,

(2) the regular horizontal trajectories form a cylinder $A$ that fills $\overline{\mathbf{C}} \backslash(E \cup \Delta(p))$ except for a critical graph $C$,

(3) each regular horizontal trajectory $\alpha$ in this cylinder has length equal to $2 \pi$ and the totality of these trajectories fill $A$,

(4) $C$ is a closed, connected set of measure zero and is the union of critical horizontal trajectories of $q$ that join its zeros and poles,

(5) $C$ is a connected, simply connected finite graph, the poles of $q$ form the endpoints of $C$ and any zero of order $k$ is a vertex of $C$ from which $k+2$ edges of $C$ emanate, and

(6) $\|q\|=\iint_{\overline{\mathbf{C}} \backslash(E \cup \Delta(p))}|q(z)| d x d y=2 \pi b$, where $b$ is the height of $A$ measured in the metric $|q|^{1 / 2}$.

$q$ is the unique holomorphic quadratic differential on $Y$ with the properties that it has a characteristic cylinder with maximal modulus among all cylinders homotopic to the boundary of $\Delta(p)$ and its circumference measured with respect to the metric $|q(z)|^{1 / 2}|d z|$ is equal to $2 \pi$.

Proof. The existence and uniqueness of $q$ with these properties is well-known, see $[30,19,15,12]$. For any simple closed curve $\alpha$ not homotopically trivial and not homotopic to a puncture on any hyperbolic Riemann surface, such a holomorphic quadratic differential is obtained by maximizing the modulus of a cylinder among all cylinders on the surface homotopic to $\alpha$. A similar conclusion is true even if we begin with a system $\left\{\alpha_{j}\right\}$ of non-homotopic simple closed curves, and either the heights of the annuli can be arbitrarily specified (the heights theorem of Renelt [27]) or the projective class of the moduli of the annuli can be arbitrarily specified (the Strebel moduli theorem [30]).

For completeness of exposition here we give a sketch of the proof in the case we need, namely, the case where there is just one annulus with core curve homotopic to a boundary component and the size of this boundary component shrinks to zero.

Lemma 1. Suppose $A$ is an annular Riemann surface conformal to the ring domain $\{z: r<|z|<R\}$ and $\mu(z)$ is an $L^{\infty}$ Beltrami differential on $A$ with $\|\mu\|_{\infty}<$ 1. In terms of the conformal parameter $w$ let $q(w)(d w)^{2}=\left(\frac{d z}{z}\right)^{2}$ and let $A_{\mu}$ be the same annulus with the conformal structure induced by $\mu$. This means a local 
homeomorphism $w=h(z)$ from a neighborhood of a point $p$ in $A$ is declared to be conformal if

$$
h_{\bar{z}}(z)=\mu(z) h_{z}(z) .
$$

Let $\Lambda(A)$ be the extremal length of the family of arcs in $A$ that join its two boundary components and $\Lambda\left(A_{\mu}\right)$ be the extremal length with the same family with respect to the conformal structure induced by $\mu$. Then

$$
\log \Lambda\left(A_{t \mu}\right)=\log \Lambda(A)+2 \operatorname{Re} \frac{t}{\|q\|} \iint_{A} \mu q d x d y+O\left(|t|^{2}\right) .
$$

Proof. This formula follows from the Reich-Strebel inequalities and is proved in [13] and [15].

Lemma 2. Suppose $A$ is an annulus embedded in a Riemann surface $Y$ and the modulus of $A$ is as large as possible among all annuli homotopic to $A$ in $Y$. Let $g$ be a conformal map from $A$ onto the region $\{z: r<|z|<R\}$. Then $-\left(\frac{d g}{g}\right)^{2}$ is the restriction of a holomorphic quadratic differential $q$ on $Y$ and

$$
\iint_{Y}|q| d x d y=\iint_{A}\left|\frac{1}{z^{2}}\right| d x d y=2 \pi \log (R / r) .
$$

All of the regular horizontal trajectories of $q$ are closed and are the images under $g$ of the circles $\rho e^{i \theta}, r<\rho<R$.

Proof. This lemma is proved in [12] and, for general measured foliations, in [16]. For the benefit of the reader we repeat the main ideas of the proof here.

The complex Banach space $Q(Y)$ of integrable holomorphic quadratic differentials on $Y$ is a closed subspace of $L^{1}(Y)$, the space of integrable quadratic differentials and the dual Banach space $L^{1}(Y)^{*}$ is isometric to $L^{\infty}$, the space of essentially bounded Beltrami differentials under the pairing

$$
(q, \mu)=\iint_{Y} \mu q d x d y
$$

In particular, if $\mu$ represents a linear functional $\ell \in L^{1}(Y)^{*}$ then

$$
\|\ell\|_{*}=\sup _{q \in L^{1}(Y)} \frac{\left(\iint \mu q d x d y\right)}{\|q\|}=\|\mu\|_{\infty} .
$$

Our strategy to prove this lemma uses the identification of $Q(Y)^{\perp \perp}$ with $Q(Y)$ provided by this pairing. Showing that $\left(\frac{d g}{g}\right)^{2}$ is in $Q(Y)^{\perp \perp}$ also shows that it is in $Q(Y)$. If $\left(\frac{d g}{g}\right)^{2} \notin Q(Y)^{\perp \perp}$, then there is a Beltrami differential $\mu$ supported in $A$ such that

$$
\iint_{A} \mu q d x d y=0
$$

for all $q \in Q(Y)$ and $\iint_{A} \mu\left(\frac{d g}{g}\right)^{2} d x d y \neq 0$. By the Hamilton-Krushkal variational lemma there would be a curve of deformations $\mu_{t}$ tangent to $\mu$ at $t=0$ for which, by Lemma $1, \Lambda\left(A_{\mu_{t}}\right)$ is smaller than $\Lambda(A)$ and for which $Y\left(\mu_{t}\right)$ represents the same point of Teichmüller space as $Y$. This conclusion contradicts the assumption that $\Lambda(A)$ has maximum modulus among homotopic annuli $A$ embedded in $Y$. 
The following result is also well known $[15,17]$.

Lemma 3. Let $f$ be a quasiconformal homeomorphism mapping a hyperbolic Riemann surface $Y$ to a Riemann surface $f(Y)$ and let $K$ be the maximal dilatation of $f$. Let $q$ be a holomorphic quadratic differential on $Y$ of finite norm with given heights and $q_{f}$ a holomorphic quadratic differential on $f(Y)$ such the height along the isotopy class of any curve $\gamma$ in $Y$ measured with respect to $\left|\operatorname{Im}\left(q(z)^{1 / 2} d z\right)\right|$ is equal to the height of the isotopy class $f(\gamma)$ in $f(Y)$ measured with respect to $\left|\operatorname{Im}\left(q_{f}(w)^{1 / 2} d w\right)\right|$. Then

$$
K^{-1}\|q\| \leq\left\|q_{f}\right\| \leq K\|q\|
$$

Proof. By the Dirichlet principle $[14,15]$ for measured foliations

$$
\|q\|=\iint_{Y}|q| d x d y
$$

is equal to the infimum of $2 D(v)=2 \iint_{Y}\left(v_{x}^{2}+2 v_{y}^{2}\right) d x d y$, where the infimum is taken over all measured foliations $|d v|$ that realize the heights of $q$, and any measured foliation that realizes this infimum is the absolute value of the imaginary part of the square root of $q$. Note that $|d(v \circ f)|$ has the same corresponding heights on $Y$ that $|d v|$ has on $f(Y)$, and

$$
(v \circ f)_{z}=\left(v_{w} \circ f\right) f_{z}+\left(v_{\bar{w}} \circ f\right) \bar{f}_{z} .
$$

Since $v$ is real-valued and defined up to plus or minus sign and up to the addition of a constant, $\left|v_{w}\right|$ and $\left|v_{\bar{w}}\right|$ are invariant and $\left|v_{w}\right|^{2}=\left|v_{\bar{w}}\right|^{2}=(1 / 4)\left(v_{x}^{2}+v_{y}^{2}\right)$. Thus we can use exactly the same calculation that is given at the end of Chapter 1 in [1]. We have

$$
\left|(v \circ f)_{z}\right| \leq\left(\left|v_{w}\right| \circ f\right)\left(\left|f_{z}\right|+\left|f_{\bar{z}}\right|\right)
$$

and

$$
\begin{aligned}
D_{Y}(|d v \circ f|) & =2 \iint_{Y}\left|(v \circ f)_{z}\right|^{2}|d z \wedge \overline{d z}| \leq 2 \iint_{Y}\left(\left|v_{w}\right| \circ f\right)^{2}\left(\left|f_{z}\right|+\left|f_{\bar{z}}\right|\right)^{2}|d z \wedge d \bar{z}| \\
& =2 \iint_{f(Y)}\left|v_{w}\right|^{2} \frac{\left(\left|f_{z}\right|+\left|f_{\bar{z}}\right|\right)^{2}}{\left|f_{z}\right|^{2}-\left|f_{\bar{z}}\right|^{2}}|d w \wedge d \bar{w}| \\
& =2 \iint_{f(Y)}\left|v_{w}\right|^{2}\left(\frac{\left|f_{z}\right|+\left|f_{\bar{z}}\right|}{\left|f_{z}\right|-\left|f_{\bar{z}}\right|}\right)|d w \wedge d \bar{w}| \leq K(f) D_{f(Y)}(|d v|) .
\end{aligned}
$$

This implies the left hand side of (3). The right hand side follows from the same argument applied to $f^{-1}$.

Lemmas 1, 2, and 3 and a limiting process yield the proof of Theorem 3.

\section{Definition of the extension}

In this section we assume the hypothesis of Theorem 1. Thus we are given a holomorphic motion

$$
E_{t}=h(t, E)=\left\{p_{1}(t)=0, p_{2}(t)=1, p_{3}(t)=\infty, p_{4}(t), \ldots, p_{n}(t)\right\}
$$

of the finite set

$$
E=\left\{p_{1}=0, p_{2}=1, p_{3}=\infty, p_{4}, \ldots, p_{n}\right\}
$$

in $\overline{\mathbf{C}}$ parameterized by the pointed hyperbolic Riemann surface $X$. 
From the guiding quasiconformal isotopy $G(t, z): X \times \overline{\mathbf{C}} \rightarrow \overline{\mathbf{C}}$, we are given another continuous function

$$
p_{n+1}(t)=G(t, p): X \rightarrow \overline{\mathbf{C}}
$$

so that

$$
\widetilde{E}_{t}=E_{t} \cup\left\{p_{n+1}(t)\right\}=\left\{p_{1}(t)=0, p_{2}(t)=1, p_{n+1}(t)=\infty, p_{4}(t), \ldots, p_{n}(t), p_{n+1}(t)\right\}
$$

is a continuous motion of the set

$$
\widetilde{E}=\left\{p_{1}=0, p_{2}=1, p_{3}=\infty, p_{4}, \ldots, p_{n}, p_{n+1}=p\right\}
$$

parameterized by $X$ and $\widetilde{E}_{t}$ is a set of $n+1$ distinct points in $\overline{\mathbf{C}}$ for each $t \in X$.

Let us use

$$
\widetilde{h}(t, z)=\left\{\begin{array}{ll}
p_{j}(t) & \text { for } z=p_{j}, 1 \leq j \leq n, \text { and } \\
p_{n+1}(t) & \text { for } z=p
\end{array}: X \times \widetilde{E} \rightarrow \overline{\mathbf{C}}\right.
$$

to denote this continuous motion. Note that it is a holomorphic motion when restricted on $X \times E$.

Among univalent functions $f$ from the open unit disc $\Delta$ into $\overline{\mathbf{C}} \backslash E$ with $f(0)=p$, by a normal families argument, one can select such a function for which $\left|f^{\prime}(0)\right|$ is as large as possible. Then $D=f(\Delta \backslash\{0\})$ is a punctured disc embedded in $\overline{\mathbf{C}} \backslash E$ with a puncture at the point $p$.

If we put $w=f^{-1}(z)$ then by Theorem 3 and a limiting argument, $w$ maps $D$ to $\{w: 0<|w|<1\}$ and

$$
-\left(\frac{d w}{w}\right)^{2}
$$

is the restriction of a global meromorphic quadratic differential on $\overline{\mathbf{C}}$, holomorphic except at the points in $\widetilde{E}$. It has a double pole at $p$, simple poles at the points of $E$ and closed regular horizontal trajectories that surround $p$ and that fill the Riemann sphere except for the points of $\widetilde{E}$ and the points of its compact critical trajectories. The critical horizontal trajectories form a connected tree with endpoints at the points of $E$. Pairwise identifications of arcs of equal angle along the circumference $\{|w|=1\}$ form its critical graph. In the generic case, when the critical graph has only three pronged singularities, there are $2(n+1)$ intervals that partition the circle $\{w:|w|=$ $1\}$. These intervals are pairwise identified so as to realize the critical graph with $n+1$ edges, where $n$ is the cardinality of $E$. For a proof of the existence of $q$ with these properties see [24] and [19]. We put $q$ equal to the negative of this quadratic differential. Thus, by definition,

$$
q=+\left(\frac{d w}{w}\right)^{2}
$$

in $D$ and $q$ is meromorphic on $\overline{\mathbf{C}}$ and holomorphic on $\overline{\mathbf{C}} \backslash \widetilde{E}$. It has simple poles at the points of $E$ and a double pole at $p$. The heights of $q$ determine the angles pointing from $p$ to the points of $E$ and pointing to the edges which are critical vertical trajectories of $q$.

Our goal is to use the guiding isotopy $G$ assumed in Theorem 1 to define a replacement $\widehat{h}$ of $\widetilde{h}$. This replacement is obtained by a limiting process in such a way 
that $\widehat{h}$ becomes a holomorphic motion of $\widetilde{E}$ parameterized by $X$ that extends the holomorphic motion $h$ of $E$ parameterized by $X$. We define $\widehat{h}$ in several steps.

Step I. For $0<\epsilon<1$ let $D_{\epsilon}=\left\{z \in \overline{\mathbf{C}}:|w|=\left|f^{-1}(z)\right| \leq \epsilon\right\}$ and

$$
Y_{\epsilon}=\overline{\mathbf{C}} \backslash\left(E \cup D_{\epsilon}\right) \text {. }
$$

Define the reflection $j_{\epsilon}$ across the (analytic) circle $\alpha_{\epsilon}=\{z:|w|=\epsilon\}$ by

$$
j_{\epsilon}(w)=\frac{\epsilon^{2}}{\bar{w}}
$$

It maps the annulus $\{z: \epsilon<|w|<1\}$ onto the annulus $\left\{z: \epsilon^{2}<|w|<\epsilon\right\}$ and we can form the double Riemann surface $Y_{\epsilon}^{d}$ of $Y_{\epsilon}$ which is

$$
Y_{\epsilon}^{d}=Y_{\epsilon} \cup \alpha_{\epsilon} \cup j_{\epsilon}\left(Y_{\epsilon}\right) \text {. }
$$

$Y_{\epsilon}^{d}$ becomes a surface of finitie analytic type by identifying arcs on the circle $\{w:|w|=$ $\left.\epsilon^{2}\right\}$ that correspond under the reflection $j_{\epsilon}$ to identified arcs on the circle $\{w:|w|=$ $1\}$.

Step II. The quadratic differential $q$ induces a quadratic differential $q_{\epsilon}$ on $Y_{\epsilon}^{d}$ by using the image under $j_{\epsilon}$ of identifications on $\{z:|w|=1\}$ as indentifications along the circle $\left\{z:|w|=\epsilon^{2}\right\}$. The resulting Riemann surface is a complex sphere with $2 n$ points removed, namely, the points of $E \cup j_{\epsilon}(E)$. On that surface the norm of $q_{\epsilon}$ is finite and given by

$$
\left\|q_{\epsilon}\right\|=\iint_{Y_{\epsilon}^{d}}\left|q_{\epsilon}\right|=2 \pi \log \left(1 / \epsilon^{2}\right)=-4 \pi \log \epsilon .
$$

The critical vertical trajectories of $q_{\epsilon}$ form two trees, the first of which has endpoints comprised of the points of $E$ and the second of which has endpoints $j_{\epsilon}(E)$. All of the zeros of $q_{\epsilon}$ are located at the interior nodes of these two trees and the poles are located at the points of $E \cup j_{\epsilon}(E)$. If $n$ is the cardinality of $E$ then the number of poles of $q_{\epsilon}$ on the Riemann sphere is $2 n$ and then number of zeros is $2 n-4$.

Note that the horizontal trajectories of $q_{\epsilon}$ are radial lines in the $w$-parameter and that for $w=r e^{i \theta}$ the vertical measure is $|d \theta|$.

Step III. By restriction the guiding isotopy $G_{t}(z)=G(t, z)$ yields a quasiconformal map

$$
G_{t, \epsilon}: Y_{\epsilon} \rightarrow Y_{t, \epsilon}=G_{t, \epsilon}\left(Y_{\epsilon}\right)
$$

Let $Y_{t, \epsilon}^{d}$ be the surface $Y_{t, \epsilon}$ doubled along its boundary curve $\alpha_{t, \epsilon}=G_{t}\left(\alpha_{\epsilon}\right)$. There is an anticonformal involution $j_{t, \epsilon}$ of $Y_{t, \epsilon}^{d}$ with the property that $Y_{t, \epsilon}^{d}$ is the union of the domains $Y_{t, \epsilon}, j_{t, \epsilon}\left(Y_{t, \epsilon}\right)$ and a simple closed curve $\alpha_{t, \epsilon}$. The curve $\alpha_{t, \epsilon}$ comprises the common boundary of these the Fuchsianization of these two domains and the involution $j_{t, \epsilon}$ fixes the points of $\alpha_{t, \epsilon}$. Thus we have

$$
Y_{t, \epsilon}^{d}=Y_{t, \epsilon} \cup \alpha_{t, \epsilon} \cup j_{t, \epsilon}\left(Y_{t, \epsilon}\right) \text {. }
$$

Without changing the notation, we let $G_{t, \epsilon}$ equal to $G_{t}$ in the domain $Y_{\epsilon}$ and equal to $j_{t, \epsilon} \circ G_{t} \circ j_{\epsilon}^{-1}$ in the domain $j_{\epsilon}\left(Y_{\epsilon}\right)$. Thus we get a quasiconformal homeomorphism

$$
G_{t, \epsilon}: Y_{\epsilon}^{d} \rightarrow Y_{t, \epsilon}^{d}
$$

Then $G_{t, \epsilon}$ induces a heights mapping from quadratic differentials on $Y_{\epsilon}^{d}$ onto quadratic differentials on $Y_{t, \epsilon}^{d}$. The heights mapping carries $q_{\epsilon}$ to a meromorphic quadratic 
differential $q_{t, \epsilon}$ with simple poles at the points of $E_{t} \cup j_{t, \epsilon}\left(E_{t}\right)=G_{t, \epsilon}(E) \cup G_{t, \epsilon}\left(j_{\epsilon}(E)\right)$ and with finite norm,

$$
\left\|q_{t, \epsilon}\right\| \leq K_{t}(-4 \pi \log \epsilon)
$$

where $K_{t}$ is the maximal dilatation of $G_{t}$.

Step IV. Now take a sequence of positive numbers $\epsilon_{n}$ decreasing to 0 and let $q_{t, n}=q_{t, \epsilon_{n}}$. For any sequence of positive integers $k \geq 1$ the quadratic differentials $q_{t, n+k}$ have common domain of definition $Y_{t, \epsilon_{n}}$ and for all $k$ one has the inequality

$$
\iint_{Y_{t, \epsilon_{n}}}\left|q_{t, n+k}\right| \leq K_{t}\left(-4 \pi \log \epsilon_{n}\right) .
$$

Now take a normal limit on compact subsets, first as $k \rightarrow \infty$ and then as $n \rightarrow \infty$, and denote the limiting quadratic differential by $q_{t}$.

Step V. The quadratic differential $q_{t}$ has the following properties:

i) $q_{t}$ is meromorphic on the Riemann sphere $\overline{\mathbf{C}}$ and holomorphic except at $n+1$ points,

ii) it has simple poles at the $n$ points of $E_{t}=G_{t}(E)$ and a double pole at another point $\widehat{p}(t)$

iii) the height of $q_{t}$ along the homotopy class of a simple curve that surrounds $\widehat{p}(t)$ is $2 \pi$, and

iv) the heights of other simple closed curves in the Riemann sphere minus $E_{t}$ are equal to the corresponding heights of $q$ on the Riemann sphere minus $E$.

\section{Step VI.}

Definition 5. We define the replacement $\widehat{h}$ of $\widetilde{h}$ by

$$
\widehat{h}(t, z)= \begin{cases}\widehat{p}_{j}(t)=\lim _{\epsilon \rightarrow 0} G_{t, \epsilon}(z) & \text { for } z=p_{j}, 1 \leq j \leq n, \text { and } \\ \widehat{p}_{n+1}(t)=\widehat{p}(t) & \text { for } z=p .\end{cases}
$$

Lemma 4. The map $\widehat{h}(t, z)$ is a motion of $\widetilde{E}$ that extends the holomorphic motion $h$ of $E$. That is, for each $t \in X$ the extension $\widehat{h}$ of $h$ is an injection from $\widetilde{E}$ into $\overline{\mathbf{C}}$.

Proof. The map $G_{t, \epsilon}$ defined in Step III depends on the reflection defined in Step II, which depends on $\epsilon$. The heights mapping induced by $G_{t, \epsilon}$ carries $q_{\epsilon}$ to a holomorphic quadratic differential $q_{t, \epsilon}$ on $Y_{t, \epsilon}^{d}$ with simple poles at $2 n$ points, $n$ of which lie in $Y_{t, \epsilon}$ and $n$ of which lie in $j_{t, \epsilon}\left(Y_{t, \epsilon}\right)$. As $\epsilon \rightarrow 0$ the location of the poles of $q_{t, \epsilon}$ in $Y_{t, \epsilon}$ converge to the points of $E_{t}$. Therefore, $\widehat{p}_{j}(t)=p_{j}(t)$ for all $1 \leq j \leq n$ and all $t \in X$. Since $\widehat{p}(t)=\widehat{h}(t, p)$ lies in $j_{t, \epsilon}\left(Y_{t, \epsilon}\right)$ and the modulus of the maximal annulus in $Y_{t, \epsilon}^{d} \backslash\left(E_{t} \cup j_{t, \epsilon}\left(Y_{t, \epsilon}\right)\right)$ increases to infinity as $\epsilon \rightarrow 0$. Thus $\widehat{p}_{n+1}(t)=\widehat{p}(t)$ cannot coincide with any of those points in $E_{t}$.

Note that although $\widehat{p}_{j}(t)=G\left(t, p_{j}\right)=p_{j}(t)$ for $1 \leq j \leq n$ and $t \in X$, the location of the point $\widehat{p}_{n+1}(t)=\widehat{h}(t, p)$ is not equal to $p_{n+1}(t)=G(t, p)$. The limiting differential

$$
q_{t}=\lim _{\epsilon \rightarrow 0} q_{t, \epsilon}
$$


is holomorphic on the Riemann sphere $\overline{\mathbf{C}}$ except at the points $\widehat{E}_{t}=E_{t} \cup\{\widehat{p}(t)\}$. It has simple poles at the points of $E_{t}$ and a double pole with quadratic residue equal to 1 at $\widehat{p}(t)$. The sense in which (11) is a limit is described in Step IV; it is a limit in the uniform topology on compact sets. In the next section we show that the location $\widehat{p}(t)$ of the double pole of $q_{t}$, which is the definition of $\widehat{h}(t, p)$, extends $h$ holomorphically to the point $p \in \overline{\mathbf{C}} \backslash E$.

\section{Harmonic coordinates and the holomorphic replacement}

For $t \in X$, let $\overline{Y_{t, \epsilon}^{d}}=\overline{\mathbf{C}}$ be the Riemann sphere such that

$$
Y_{t, \epsilon}^{d}=\overline{Y_{t, \epsilon}^{d}} \backslash\left(E_{t} \cup j_{t, \epsilon}\left(E_{t}\right)\right) .
$$

Remember we always assume that $0,1, \infty$ are in $E_{t}$.

Consider the Teichmüller space Teich $\left(Y_{t, \epsilon}^{d}\right)$. Let $\mathcal{M}\left(Y_{t, \epsilon}^{d}\right)$ be the space of all Beltrami differentials $\mu$ on $Y_{t, \epsilon}^{d}$. That is, $\mu=\mu(z) d \bar{z} / d z$ such that $\mu(z) \in L^{\infty}\left(Y_{t, \epsilon}^{d}\right)$ with $\|\mu(z)\|_{\infty}<1$. Let $w^{\mu}$ be the quasiconformal homeomorphism fixing $0,1, \infty$ solving the Beltrami equation

$$
w_{\bar{z}}=\mu(z) w_{z} .
$$

Then two elements $\mu, \nu \in \mathcal{M}\left(Y_{t, \epsilon}^{d}\right)$ are equivalent if $\left(w^{\nu}\right)^{-1} \circ w^{\mu}$ is homotopic to the identity relative to $E_{t} \cup j_{t, \epsilon}\left(E_{t}\right)$ and the Techmüller space Teich $\left(Y_{t, \epsilon}^{d}\right)$ is the space of all equivalence classes $[\mu]$. Then we have a holomorphic spilt submersion

$$
P_{t, \epsilon}(\mu)=[\mu]: \mathcal{M}\left(Y_{t, \epsilon}^{d}\right) \rightarrow \operatorname{Teich}\left(Y_{t, \epsilon}^{d}\right) .
$$

From the Ahlfors-Weill extension procedure (see [2]), $P_{t, \epsilon}$ has a local holomorphic section

$$
S_{t, \epsilon}: U_{t, \epsilon}\left(\subset \operatorname{Teich}\left(Y_{t, \epsilon}^{d}\right)\right) \rightarrow \mathcal{M}\left(Y_{t, \epsilon}^{d}\right)
$$

defined on a neighborhood $U_{t, \epsilon}$ about the basepoint in Teich $\left(Y_{t, \epsilon}^{d}\right)$ such that

$$
P_{t, \epsilon} \circ S_{t, \epsilon}=\text { Identity. }
$$

Now consider the subspace $Y_{2 n}$ of $\overline{\mathbf{C}}^{2 n}$,

$$
Y_{2 n}=\left\{\mathbf{z}=\left(z_{1}, z_{2}, z_{3}, z_{4}, \cdots, z_{n}, z_{n+1}, \cdots, z_{2 n}\right) \in \overline{\mathbf{C}}^{2 n}\right\}
$$

where $z_{1}=0, z_{2}=1, z_{3}=\infty$, and $z_{i} \neq z_{j}$ for all $1 \leq i \neq j \leq 2 n$. Then the map

$$
\pi_{t, \epsilon}([\mu])=\left(w^{\mu}\left(E_{t}\right), w^{\mu}\left(j_{t, \epsilon}\left(E_{t}\right)\right)\right): \operatorname{Teich}\left(Y_{t, \epsilon}^{d}\right) \rightarrow Y_{2 n}
$$

is a universal holomorphic cover (see $[5,26]$ ).

Now let $t \in X$ be a fixed point and let $s \in X$ be a variable. Then the map

$$
G_{s, \epsilon} \circ G_{t, \epsilon}^{-1}: Y_{t, \epsilon}^{d} \rightarrow Y_{s, \epsilon}^{d}
$$

can be considered as a point $\left[\mu_{t, s, \epsilon}\right]$ in Teich $\left(Y_{t, \epsilon}^{d}\right)$, where $\mu_{t, s, \epsilon}$ is the Beltrami coefficient of $G_{s, \epsilon} \circ G_{t, \epsilon}^{-1}$.

Suppose $\delta>0$ is a small number. Let

$$
\Delta_{t}=\{s|| s-t \mid<\delta\}
$$

be a disk in $X$, where $|\cdot|$ means the hyperbolic distance on $X$. Then $\Delta_{t}$ is simply connected. 
Consider the map

$$
f_{\epsilon}(s)=\left(w^{\mu t, s, \epsilon}\left(E_{t}\right), j_{t, \epsilon}\left(E_{t}\right)\right)=\left(E_{s}, j_{t, \epsilon}\left(E_{t}\right)\right) .
$$

For a fixed $\delta$ small enough (depending on $t$ ), $f_{\epsilon}(s) \in Y_{2 n}$ for all $s \in \Delta_{t}$.

From the assumption that $E_{s}=w^{\mu t, s, \epsilon}\left(E_{t}\right)$ is a holomorphic motion, $f_{\epsilon}(s)$ depends on $s$ holomorphically. Since $\Delta_{t}$ is simply connected, we can lift $f_{\epsilon}(s)$ to a holomorphic map

$$
\widetilde{f}_{\epsilon}(s): \Delta_{t} \rightarrow \operatorname{Teich}\left(Y_{t, \epsilon}^{d}\right)
$$

such that

$$
P_{t, \epsilon} \circ \tilde{f}_{\epsilon}(s)=f_{\epsilon}(s) .
$$

When $\delta>0$ is small enough we have that $\tilde{f}_{\epsilon}\left(\Delta_{t}\right) \subset U_{t, \epsilon}$. Thus we get another holomorphic map

$$
\widehat{f}_{\epsilon}(s)=S_{t, \epsilon} \circ \tilde{f}_{\epsilon}(s): \Delta_{t} \rightarrow \mathcal{M}\left(Y_{t, \epsilon}^{d}\right)
$$

shown in the following diagram:

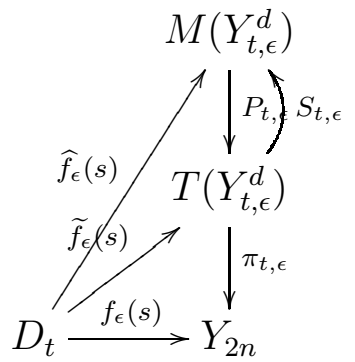

The Riemann surface

$$
\widehat{Y}_{s, \epsilon}^{d}=w^{\widehat{f}_{\epsilon}(s)}\left(Y_{t, \epsilon}^{d}\right)
$$

is the gluing of two Riemann surfaces $Y_{s, \epsilon}$ and $j_{t, \epsilon}\left(Y_{t, \epsilon}\right)$ at same angles measured by the quadratic differentials $q_{s, \epsilon}$ and $q_{t, \epsilon}$ from the heights mappings, respectively, along $\alpha_{s, \epsilon}$ and $\alpha_{t, \epsilon}$. Remember that $Y_{s, \epsilon}^{d}$ is the gluing of two Riemann surfaces $Y_{s, \epsilon}$ and $j_{s, \epsilon}\left(Y_{s, \epsilon}\right)$ at same angles measured by the quadratic differential $q_{s, \epsilon}$ from the heights mapping along the same $\alpha_{s, \epsilon}$. Note that $\alpha_{t, \epsilon}$ and $\alpha_{s, \epsilon}$ have the same total length $2 \pi$.

For any fixed $s \in D_{t}$, when $\epsilon \rightarrow 0$, both $j_{t, \epsilon}\left(Y_{t, \epsilon}\right)$ in $\widehat{Y}_{s, \epsilon}^{d}$ and $j_{s, \epsilon}\left(Y_{s, \epsilon}\right)$ in $Y_{s, \epsilon}^{d}$ considered as sets in the Riemann sphere $\overline{\mathbf{C}}$ tend to the same point $\widehat{p}(s)$. Since $w^{\widehat{f}_{\epsilon}(s)}(z)$ is holomorphic on $\Delta_{t}$ for all $\epsilon>0$, its limiting function

$$
\widehat{p}(s)=\lim _{\epsilon \rightarrow 0} w^{\widehat{f}_{\epsilon}(s)}(z), \quad \forall z \in j_{t, \epsilon}\left(Y_{t, \epsilon}\right)
$$

is also holomorphic on $\Delta_{t}$.

Finally we have shown that $\widehat{p}(t): X \rightarrow \overline{\mathbf{C}}$ is holomorphic and this completes the proof of Theorem 1 .

\section{A new proof of Slodkowski's Theorem}

In this section, we give a new proof of Theorem 2. It is based on Theorem 1 and Lemma 5 below which guarantees that when $X$ is the unit disk $\Delta$ there is always a guiding quasiconformal isotopy.

Given a finite subset $E$ in $\overline{\mathbf{C}}$ containing $0,1, \infty$. We recall some facts from the beginning of the previous section. Let $\Omega=\overline{\mathbf{C}} \backslash E$ be the Riemann surface and let $T(\Omega)$ be its Teichmüller space. Let $\mathcal{M}(\mathbf{C})$ be the open unit ball of the space $L^{\infty}(\mathbf{C})$. Each 
element $\mu \in \mathcal{M}(\mathbf{C})$ is called a Beltrami coefficient. Let $w^{\mu}$ be the quasiconformal homeomorphism fixing 0,1, $\infty$ solving the Beltrami equation (12).

Two elements $\mu_{0}, \mu_{1} \in M(\mathbf{C})$ are equivalent if there is a continuous curve of Beltrami coefficients $\mu_{t}$ coinciding with $\mu_{0}$ and $\mu_{1}$ at $t=0$ and $t=1$ such that $w^{\mu_{t}}\left|E=w^{\mu_{0}}\right| E=w^{\mu_{1}} \mid E$ for all $0 \leq t \leq 1$. We use $[\mu]_{E}$ to denote an equivalence class. The Teichmüller space $T(\Omega)$ is equal to the space $T(E)$ of all equivalent classes $[\mu]_{E}$ for all $\mu \in \mathcal{M}(\mathbf{C})$. Then $T(E)$ is a complex Banach manifold and the projection

$$
P_{E}(\mu)=[\mu]_{E}: \mathcal{M}(\mathbf{C}) \rightarrow T(E)
$$

is holomorphic.

Lemma 5. Suppose $\Delta$ is the open unit disk with the base point 0. Suppose

$$
E=\left\{p_{1}=0, p_{2}=1, p_{3}=\infty, p_{4}, \ldots, p_{n}\right\}, \quad n \geq 3,
$$

is a finite subset of the Riemann sphere $\overline{\mathbf{C}}$ and $h(t, z): \Delta \times E \rightarrow \overline{\mathbf{C}}$ is a normalized holomorphic motion of $E$. Then $h$ has a guiding quasiconformal isotopy $G(t, z): \Delta \times$ $\overline{\mathbf{C}} \rightarrow \overline{\mathbf{C}}$.

Proof. Let

$$
Y_{n-3}=\left\{\mathbf{z}=\left(z_{1}, \cdots, z_{n-3}\right) \in \mathbf{C}^{n-3}\right\}
$$

where $z_{i} \neq z_{j}$ for all $1 \leq i \neq j \leq n-3$ and $z_{i} \neq 0,1, \infty$ for all $1 \leq i \leq n-3$. From the normalized holomorphic motion $h(t, z): \Delta \times E \rightarrow \overline{\mathbf{C}}$, we can define a holomorphic map

$$
f(t)=\left(h\left(t, p_{4}\right), \cdots, h\left(t, p_{n}\right)\right): \Delta \rightarrow Y_{n-3} .
$$

We know that the map

$$
\pi_{E}\left([\mu]_{E}\right)=\left(w^{\mu}\left(p_{4}\right), \cdots, w^{\mu}\left(p_{n}\right)\right): T(E) \rightarrow Y_{n-3}
$$

is a holomorphic universal covering (refer to $[5,26]$ ). Since $\Delta$ is simply connected, we can lift $f$ to get a holomorphic map

$$
\widetilde{f}(t): \Delta \rightarrow T(E)
$$

such that

$$
\pi_{E} \circ \tilde{f}=f
$$

From the Douady-Earle barycentric extension procedure (see [6]), there is a continuous section $S$ of $P_{E}$ (see [20]), that is, a continuous map $S$ from $T(E)$ to $M(\mathbf{C})$ such that $P_{E} \circ S$ is the identity on $T(E)$. Define

$$
\widehat{f}(t)=S \circ \tilde{f}(t): \Delta \rightarrow M(\mathbf{C})
$$

Then we have that

$$
P_{E} \circ \widehat{f}=\widetilde{f} \quad \text { and } \quad \pi_{E} \circ P_{E} \circ \widehat{f}=f
$$


The relationship of the various maps is illustrated in the diagram below, which is the same as the diagram shown in the previous section except for different labeling.

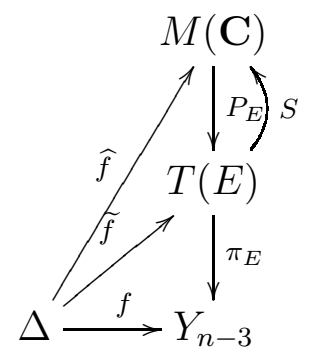

For any $\widehat{f}(t) \in M(\mathbf{C})$, Define

$$
G(t, z)=w^{\widehat{f}(t)}(z): \Delta \times \overline{\mathbf{C}} \rightarrow \overline{\mathbf{C}} .
$$

Since $\pi_{E} \circ P_{E}(\widetilde{f})=f$, we get $G(t, 0)=0, G(t, 1)=1, G(t, \infty)=\infty$, and

$$
\left(G\left(t, p_{4}\right), \cdots, G\left(t, p_{n}\right)\right)=f(t) .
$$

Thus $G$ is an extension of $h$. Thus $G$ is a guiding quasiconformal isotopy for $h$. This completes the proof.

Lemma 5 says that for any normalized holomorphic motion $h: \Delta \times E \rightarrow \overline{\mathbf{C}}$ of any finite subset $E$ in $\overline{\mathbf{C}}$ parameterized by $\Delta$, our guiding quasiconformal isotopy assumption in Theorem 1 holds. Thus for any new point $p \in \overline{\mathbf{C}} \backslash E$, we can have a holomorphic motion $\widehat{h}: \Delta \times(E \cup\{p\}) \rightarrow \overline{\mathbf{C}}$ extending $h$. A general version of Lemma 5 is proved as [25, Theorem C] and [20, Theorem 5.5]. To complete the proof, we need the $\lambda$-Lemma of Mañé, Sad and Sullivan, [23].

Lemma 6. ( $\lambda$-Lemma) Suppose $h(t, z): \Delta \times E \rightarrow \overline{\mathbf{C}}$ is a holomorphic motion, where $E$ is a (not necessarily finite) subset of $\overline{\mathbf{C}}$. Then it can be extended uniquely to a holomorphic motion $\bar{h}(t, z): \Delta \times \bar{E} \rightarrow \overline{\mathbf{C}}$, where $\bar{E}$ means the closure of $E$ in $\overline{\mathbf{C}}$.

Now suppose $h(t, z): \Delta \times E \rightarrow \overline{\mathbf{C}}$ is the normalized holomorphic motion in Theorem 2. Let $E_{\infty}=\left\{0,1, \infty, p_{1}, \cdots, p_{n}, \cdots\right\}$ be a countable dense subset of $E$. Let $F=$ $\left\{q_{1}, \cdots, q_{n}, \cdots\right\}$ be a countable dense subset of $\overline{\mathbf{C}} \backslash E$. Let $E_{n}=\left\{0,1, \infty, p_{1}, \cdots, p_{n}\right\}$ and $F_{n}=\left\{q_{1}, \cdots, q_{n}\right\}$. Then $h_{n}=h \mid \Delta \times E_{n}$ is a holomorphic motion for every $n>3$. Our main result (Theorem 1) with the consideration of Lemma 5 implies that we can extend $h_{n}$ to a holomorphic motion $H_{n}(t, z): \Delta \times\left(E_{n} \cup F_{n}\right) \rightarrow \overline{\mathbf{C}}$. Inductively, we have a holomorphic motion $H_{\infty}(t, z): \Delta \times\left(E_{\infty} \cup F\right) \rightarrow \overline{\mathbf{C}}$ which extends every $h_{n}$. The $\lambda$-Lemma implies we can extend this last holomorphic motion into a holomorphic motion $H(t, z)$ of the closure of $E_{\infty} \cup F$ which is the whole Riemann sphere $\overline{\mathbf{C}}$ with parameter space $\Delta$. This holomorphic motion $H$ is an extension of $h$ and this completes our new proof of Theorem 2 .

\section{Trace monodromy and the isotopy principle}

In this section we show why the guiding isotopy assumption in Theorem 1 is necessary. To do this we describe a topological obstruction to the extension of continuous motions parameterized by a surface with non-trivial fundamental group. This description depends on monodromy and something we call trace monodromy, concepts which are developed in [3]. Using these ideas we give an example of a holomorphic motion of a finite subset in the Riemann sphere parameterized by any non-simply 
connected bounded domain in the complex plane that cannot be extended to a continuous motion of the whole Riemann sphere parameterized by the same domain.

Suppose $X$ is any hyperbolic Riemann surface and $E=\left\{0,1, \infty, p_{4}, \ldots, p_{n}\right\}$. If $h$ is a continuous motion of $X \times E \rightarrow \overline{\mathbf{C}}$, then for any $z$ in $E-\{0,1, \infty\}, h(t, z)=$ $h^{z}(t): X \rightarrow \mathbf{C}_{0,1}=\overline{\mathbf{C}} \backslash\{0,1, \infty\}$ is continuous and, for any choice of $z \in E-\{0,1, \infty\}$ one obtains a homomorphism of fundamental groups:

$$
h_{*}^{z}: \pi_{1}(X) \rightarrow \pi_{1}\left(\mathbf{C}_{0,1}\right) .
$$

We call these homomorphisms the trace monodromies induced by $h$. Taking into account the arbitrary normalization at three points in $E$ and the arbitrary choice of a fourth, one finds that the number of different trace monodromy conditions is given by the binomial coefficient

$$
\left(\begin{array}{l}
n \\
4
\end{array}\right)
$$

By definition a trace monodromy is trivial if it maps every element of $\pi_{1}(X)$ to the identity of $\pi_{1}\left(\mathbf{C}_{0,1}\right)$. The trace monodromy obstruction to topological extension described in the following theorem and the more general monodromy obstruction are presented in [3].

Theorem 4. Suppose $X$ is a Riemann surface with a base point $t_{0}$. Let $h: X \times$ $E \rightarrow \overline{\mathbf{C}}$ be a normalized holomorphic motion of a finite set $E$ with $\operatorname{card}(E) \geq 4$. If $h$ has a guiding quasiconformal isotopy, then for each $z \in E$ the trace monodromy $h_{*}^{z}: \pi_{1}\left(X, t_{0}\right) \rightarrow \pi_{1}\left(\mathbf{C}_{0,1}\right)$ is trivial.

Proof. We outline the proof here. Suppose $H(t, z): X \times \overline{\mathbf{C}} \rightarrow \overline{\mathbf{C}}$ is a guiding isotopy for $h$. Let $\gamma(s), 0 \leq s \leq 1$, be a simple closed curve in $X$ with $\gamma(0)=$ $\gamma(1)=t_{0}$. Let $\mu(s)$ be the Beltrami coefficient of $H(\gamma(s), \cdot)$ which is continuous on $s$. Then $H(\gamma(s), \cdot)=w^{\mu(s)}(\cdot)$ since both are quasiconformal maps fixing $0,1, \infty$ with the same Beltrami coefficient. Let $\hat{H}(s, t)=w^{t \mu(s)}(z):[0,1] \times[0,1] \rightarrow \mathbf{C}_{0,1}$ for any $z \neq 0,1, \infty \in E$. Then it is a continuous map for which $\hat{H}(s, 1)=H(\gamma(s), z)$ and $\hat{H}(s, 0)=z$. Thus $\hat{H}(\gamma(s), z)$ is a continuous curve in $\mathbf{C}_{0,1}$ homotopic to a point $z$ in $\mathbf{C}_{0,1}$. This implies that the trace monodromy is trivial.

Using this theorem, we can construct a counterexample of a holomorphic motion of a finite subset of the Riemann sphere parameterized by any non-simply connected planar domain which does not satisfy our guiding quasiconformal isotopy assumption in Theorem 1.

Example 1. Suppose $X$ is a planar domain in the Riemann sphere $\overline{\mathbf{C}}$ such that $\overline{\mathbf{C}}-X$ has more than one connected component and at least one component contains more than one point. Let $t_{0}$ be the base point of $X$. Then for any finite subset $E$ in $\overline{\mathbf{C}}$ with $\#(E) \geq 4$, there is a holomorphic motion $h(t, z): X \times E \rightarrow \overline{\mathbf{C}}$ that has no guiding quasiconformal isotopy.

Proof. Since $\overline{\mathbf{C}}-X$ has a component containing more than one point, we can use two points in this component and a square root map to map $X$ into a halfplane. Then applying a Möbius transformation, we can assume that $X$ is a bounded planar domain such that $\overline{\mathbf{C}}-X$ has one unbounded component and several bounded components. 
Suppose $z_{0} \in E-\{0,1, \infty\}$. Since $X$ is planar, we can map it conformally to a planar domain $\widetilde{X}$ containing only one point $z_{0}$ in $E$. Thus we have a domain $\widetilde{X}$ such that $z_{0} \in \widetilde{X}$ and $\widetilde{X} \cap\left(E-\left\{z_{0}\right\}\right)=\emptyset$ and 0 is in a bounded component of $\overline{\mathbf{C}}-\widetilde{X}$ and $E-\left\{0, z_{0}\right\}$ are all in the unbounded component of $\overline{\mathbf{C}}-\widetilde{X}$ and a conformal map $z=\phi(t): X \rightarrow \widetilde{X}$ such that $\phi\left(t_{0}\right)=z_{0}$. Define $h(t, z)=z$ for any $z \neq z_{0}$ and $t \in X$ and $\phi\left(t, z_{0}\right)=\phi(t)$. Then $h$ is a holomorphic motion with non-trivial trace monodromy. From Theorem 4 , it does not have a guiding quasiconformal isotopy.

Remark 1. When the cardinality of $E$ is 4 and $X=\mathbf{C}-\{0,1\}$ is the thricepunctured sphere with a base point $t_{0}$, Douady constructed the following counterexample. Let $E=\left\{0,1, \infty, t_{0}\right\}$, let $h(t, z): X \times E \rightarrow \overline{\mathbf{C}}$ and define $h$ by $h(t, 0)=0$, $h(t, 1)=1$, and $h(t, \infty)=\infty$, and $h\left(t, t_{0}\right)=t$. Then Douady showed that $h$ is a maximal holomorphic motion and, therefore, cannot be extended further. Since an annulus $A$ can be thought as a covering space of the thrice-punctured sphere, there is a covering map $\pi: A \rightarrow X$. Earle considered $\widetilde{h}(t, z)=\left(\pi^{*} h\right)(t, z)=h(\pi(t), z): A \times E \rightarrow \overline{\mathbf{C}}$ and showed it is a maximal holomorphic motion and so it also cannot be extended further. See [8] for these two counterexamples and the definition of a maximal holomorphic motion. The topological obstruction defined in [3] gives us more flexibility to construct more counterexamples. One can find other counterexamples when the parameter space is the punctured disk in [3] or an annulus.

Remark 2. In [3] it is shown that when $\operatorname{card}(E)=4$ a holomorphic motion $h: X \times E \rightarrow \overline{\mathbf{C}}$ can be extended to a holomorphic motion $\widetilde{h}: X \times \overline{\mathbf{C}} \rightarrow \overline{\mathbf{C}}$ if, and only if, its trace monodromy is trivial.

\section{References}

[1] Ahlfors, L. V.: Lectures on quasiconformal mapping. - Univ. Lecture Ser. 38, Amer. Math. Soc., 2006.

[2] Ahlfors, L. V., and G. Weill: A uniqueness theorem for Beltrami equations. - Proc. Amer. Math. Soc. 13, 1962, 975-978.

[3] Beck, M., Y. Jiang, S. Mitra, and H. Shiga: Extending holomorphic motions and monodromy. - Ann. Acad. Sci. Fenn. Math. 37, 2012, 53-67.

[4] Bers, L.: A non-standard integral equation with applications to quasiconformal mapping. Acta Math. 116, 1966, 113-134.

[5] Bers, L., and H. Royden: Holomorphic families of injections. - Acta Math. 157, 1986, 259286.

[6] Doundy, A., and C. J. EArle: Conformally natural extension of homeomorhisms of the circle. - Acta Math. 157, 1986, 23-48.

[7] EARle, C. J.: Some maximal holomorphic motions. - In: Lipa's Legacy: Proceedings of the Bers Colloquium, Contemp. Math. 211, Amer. Math. Soc., 1997, 183-192.

[8] Earle, C. J., F. P. Gardiner, and N. LAKIC: Vector fields for holomorphic motions of closed sets. - In: Lipa's Legacy: Proceedings of the Bers Colloquium, Contemp. Math. 211, Amer. Math. Soc., 1997, 193-225.

[9] Earle, C. J., I. Kra, and S. L. Krushkal: Holomorphic motions and Teichmüller spaces. Trans. Amer. Math. Soc. 343, 1994, 927-948.

[10] Earle, C. J., and C. T. McMullen: Quasiconformal isotopies. - In: Holomorphic Functions and Moduli I, 1988, 143-154. 
[11] Earle, C. J., and S. Mitra: Variation of moduli under holomorphic motions - In: Contemporary Mathematics, frm-e56, 2000, 39-67.

[12] Gardiner, F.P.: The existence of Jenkins-Strebel differentials from Teichmüller theory. Amer. J. Math. 99, 1975, 1097-1104.

[13] Gardiner, F. P.: Schiffer's interior variation and quasiconformal mappings. - Duke Math. J. 42, 1975, 371-380.

[14] Gardiner, F. P.: Measured foliations and the minimal norm property for quadratic differentials. - Acta Math. 152, 1984, 57-76.

[15] Gardiner, F. P.: Teichmüller theory and quadratic differentials. - John Wiley \& Sons, New York, 1987.

[16] Gardiner, F. P., and N. Lakic: Quasiconformal Teichmüller theory. - Amer. Math. Soc., Providence, Rhode Island, 2000.

[17] Gardiner, F. P., and H. Masur: Extremal length geometry of Teichmüller space. - Complex Variables 16, 1991, 209-237.

[18] Hubbard, J., and H. Masur: Quadratic differentials and foliatons. - Acta Math. 142, 1979, $221-274$.

[19] Jenkins, J. A.: On the existence of certain general extremal metrics. - Ann. of Math. 66, 1957, $440-453$.

[20] Jiang, Y., and S. Mitra: Douady-Earle section, holomorphic motions, and some applications. - Contemp. Math. 575, Amer. Math. Soc., 2012, 219-252.

[21] Leutbecher, A.: Über Spitzen diskontinuierlichen Gruppen von lineargebrochenen Transformationen. - Math. Z. 100, 1967, 183-200.

[22] Lieb, G. S.: Holomorphic motions and Teichmüller space. - Ph.D. dissertation, Cornell University, 1990.

[23] Mañé, R., P. Sad, and D. Sullivan: On the dynamics of rational maps. - Ann. Sci. Èc. Norm. Sup. 96, 1983, 193-217.

[24] Minda, C. D.: The Hahn metric on Riemann surfaces. - Kodai Math. J., 6, 1983, 57-69.

[25] Mitra, S.: Teichmüller spaces and holomorphic motions. - J. Anal. Math. 81, 2000, 1-33.

[26] NAG, S.: The Torelli spaces of punctured tori and spheres. - Duke Math. J. 48, 1981, 359-388.

[27] Renelt, H.: Konstruktion gewisser quadratischer Differential mit von Dirichletintergralen. Math. Nachr. 73, 1976, 125-142.

[28] Shimizu, H.: On discontinuous groups operating on the product of upper half-planes. - Ann. Math. 77, 1959, 33-77.

[29] SLodkowski, Z.: Holomorphic motions and polynomial hulls. - Proc. Amer. Math. Soc. 111, $1991,347-355$.

[30] Strebel, K.: Quadratic differentials. - Springer-Verlag, Berlin \& New York, 1984.

[31] Sullivan, D. P., and W. P. Thurston: Extending holomorphic motions. - Acta Math. 157, $1986,243-257$. 